Forum Kind Jugend Sport 2020 1 1:100-109 https://doi.org/10.1007/s43594-020-00016-3 Online publiziert: 17 . November 2020 (c) Deutsche Sportjugend im DOSB e.V. 2020

Oliver Kauer-Berk' - Ulrike Burrmann ${ }^{2}$ - Ahmet Derecik - Petra Gieß-Stüber $^{4}$. Detlef Kuhlmann ${ }^{5}$. Nils Neuber ${ }^{6}$. Alfred Richartz ${ }^{7}$. Bettina Rulofs ${ }^{8}$. Jessica Süßenbach ${ }^{9}$ Ralf Sygusch ${ }^{10}$

${ }^{1}$ Redaktion Forum Kinder- und Jugendsport, Gelnhausen, Deutschland

${ }^{2}$ Sportpädagogik, Humboldt-Universität zu Berlin, Berlin, Deutschland

${ }^{3}$ Erziehungs- und Kulturwissenschaften, Universität Osnabrück, Osnabrück, Deutschland

${ }^{4}$ Sportpädagogik, Albert-Ludwigs-Universität Freiburg, Freiburg, Deutschland

${ }^{5}$ Sport und Erziehung, Leibniz Universität Hannover, Hannover, Deutschland

${ }^{6}$ Bildung und Unterricht im Sport, Westfälische Wilhelms-Universität Münster, Münster, Deutschland

${ }^{7}$ Bewegungs- und Sportpädagogik, Universität Hamburg, Hamburg, Deutschland

${ }^{8}$ Sportsoziologie, Bergische Universität Wuppertal, Wuppertal, Deutschland

${ }^{9}$ Sportpädagogik, Leuphana Universität Lüneburg, Lüneburg, Deutschland

${ }^{10}$ Sportpädagogik, Friedrich-Alexander-Universität Erlangen-Nürnberg, Erlangen, Deutschland

\title{
Das Virus, der Sport und die Herausforderungen
}

\section{Fragen an die Wissenschaft}

\section{Welche Herausforderungen bringt die Covid-19-Pandemie für den Kinder- und Jugendsport mit sich? Forum Kinder- und Jugendsport fragte dazu neun Wissenschaftler*innen im Forschungsverbund der Deutschen Sportjugend.}

\section{„Soziale Ungleichheiten im Sport diskutieren"}

\section{Forschungsthema: Integration im Kinder- und Jugendsport}

\section{Prof. Dr. Ulrike Burrmann}

Welche Auswirkungen hat die Covid19-Pandemie auf das Aufwachsen und das Sportengagement von Kindern und Jugendlichen aus Ihrer Forschungsperspektive? Deutschland hatte in den ersten Monaten der Pandemie im Vergleich zu vielen anderen Ländern weniger harte Einschnitte zu verzeichnen, unter anderem was Bewegungseinschränkungen im Freien und die (Dauer der) Schließung von Einrichtungen anbelangt. Dennoch konnten organisierte sportliche Aktivitäten mit Gleichaltrigen in der Schule, im Verein oder in Einrichtungen der offenen Kinder- und Jugendarbeit längere Zeit gar nicht und gegenwärtig nur mit Auf- lagen durchgeführt werden. Und auch informelle Sport- und Bewegungsaktivitäten waren möglicherweise durch den eingeschränkten Zugang beziehungsweise Schließung von Sportplätzen, öffentlichen Sporträumen oder Parkanlagen tangiert. Die negativen Auswirkungen zum Beispiel der Wegfall von regelmäßigen, strukturierten, gemeinschaftlichen Sport- und Bewegungsaktivitäten einschließlich der in diesem Rahmen stattfindenden informellen und nonformalen Bildungsprozesse - dürften Kinder aus weniger gut situierten Familien deutlicher spüren als Kinder aus gut gebildeten und einkommensstarken Elternhäusern. Die im Bildungsbereich geführten Diskussionen um die Verschärfung sozialer Ungleichheiten müssen also auf den Freizeit- und Sportbereich ausgeweitet werden.

Was weiß die Wissenschaft bis jetzt, welche Befunde kennen Sie zu den Auswirkungen der Corona-Krise? Während einige empirische Studien unter anderem zum (veränderten) Bewegungsverhalten und zu gesundheitlichen Parametern von Jugendlichen und Erwachsenen auf den Weg gebracht wurden und erste Befunde vorliegen (zum Beispiel Ammar et al. 2020; Mutz und Gerke 2020), mangelt es an entsprechenden Befunden für das Kindesalter. Das betrifft auch Fragen der Integration und sozialen Ungleichheit. Einiges deutet darauf hin, dass Heranwachsende während der Zeit der Schulund Vereinsschließungen weniger körperlich aktiv waren, mehr sitzenden Aktivitäten nachgingen und ein weniger konsistentes Schlafmuster aufwiesen als vor dieser Zeit (• Abb. 1; Guan et al. 2020). Ein Vergleich von Altersgruppen deutet allerdings darauf hin, dass 14- bis 29-Jährige im Vergleich zu den 30- bis 64-Jährigen und den 65+ ihre Sport- und Bewegungsaktivitäten deutlich seltener reduzierten (Mutz und Gerke 2020). Bei Kindern scheinen Bewegungsaktivitäten zugenommen zu haben, während Sport und Sportangebote abgenommen haben (Woll 2020a). Wenngleich auch hier Differenzierungen notwendig sind, denn Bewegungs- und Sportaktivitäten dürften von individuellen (zum Beispiel Motivation), sozialen (zum Beispiel Unterstützung der Eltern), infrastrukturellen (zum Beispiel Wohnumgebung) und sozialstrukturellen (zum Beispiel Bildung und Einkommen der Eltern) Merkmalen abhängen.

Welchen Bedarf an Forschung sehen Sie, wo sollten Schwerpunkte gesetzt 


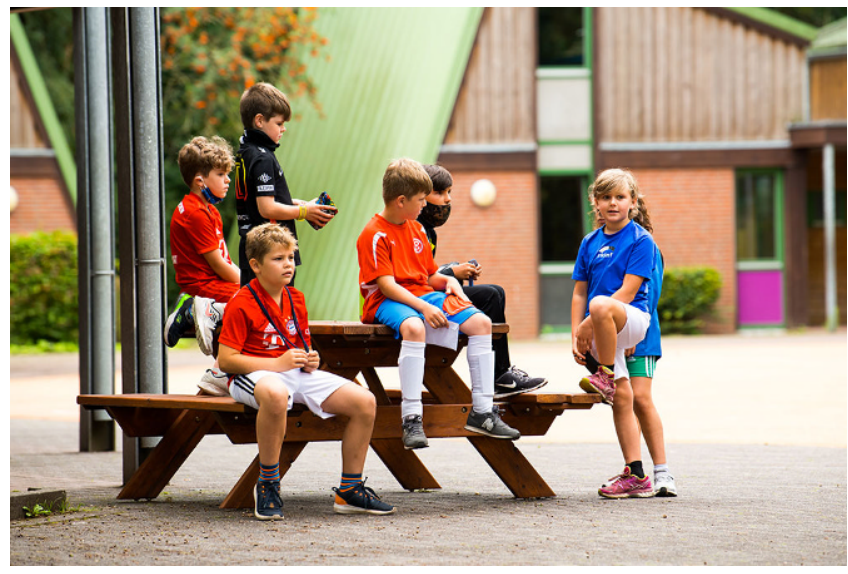

Abb. 1 A Einiges deutet darauf hin, dass Heranwachsende während der Zeit der Schul- und Vereinsschließungen weniger körperlich aktiv waren, mehr sitzenden Aktivitäten nachgingen und ein weniger konsistentes Schlafmuster aufwiesen als vor dieser Zeit. (Foto: LSBNRW/Andrea Bowinkelmann)

werden und welche Ansätze ergeben sich für Sie persönlich aus der Pandemie? Inzwischen sind unter anderem durch Fördermittel für die Corona-Forschung einige empirische Studien auch zu Fragen des Kinder- und Jugendsports auf den Weg gebracht worden. Da die Krise sehr unterschiedlich auf Personen wirkt, wäre $\mathrm{zu}$ wünschen, dass einige Studien längsschnittlich angelegt sind. Unterschiede im Sportengagement und dessen Auswirkungen auf psychosoziale Merkmale sollten differenziert analysiert werden, um die langfristigen Folgen der Pandemie einfangen und entsprechende Interventionsmaßnahmen wissenschaftlich begleiten zu können.

Mich interessieren unter anderem die Auswirkungen der Pandemie auf das freiwillige Engagement in Sportvereinen sowie Fragen des sozialen Zusammenhalts. Aus einer bundesweiten Umfrage, die allerdings vor der Pandemie und bei Erwachsenen durchgeführt wurde, geht zum Beispiel hervor, dass Mitglieder von Freiwilligenvereinigungen wie den Sportvereinen im Vergleich zu Nichtmitgliedern ein höheres $\mathrm{Maß}$ an Vertrauen gegenüber In-Groups und Out-Groups aufweisen und die gegenwärtige Gesellschaft als solidarischer und vertrauensvoller einschätzen (Burrmann et al. 2020). Ob und welche Veränderungen sich durch die Corona-Krise ergeben haben, ist offen.

Zeitpunkt der Antwort: 3. September 2020 .

\section{„Zwei neue Trends"}

\section{Forschungsthema: Partizipation im Kinder- und Jugendsport}

\section{Prof. Dr. Ahmet Derecik}

Welche Auswirkungen hat die Covid19-Pandemie auf das Aufwachsen und das Sportengagement von Kindern und Jugendlichen aus Ihrer Forschungsperspektive? Die Auswirkungen der Covid19-Pandemie scheinen auf das Aufwachsen und das Sportengagement von Kindern und Jugendlichen ambivalent auszufallen. Der organisierte Sport in Schule, Verein und bei kommerziellen Sportanbietern ist zwischenzeitlich vollständig zum Erliegen gekommen. Dennoch ist es aufgrund zwei neuer Trends in der Krisensituation nicht zwangsläufig zu verminderter Bewegung gekommen. Zum einen ist das Maß an Sport innerhalb der Familie - vor allem im Freien - gestiegen. Heranwachsende sind inzwischen mit ihren Eltern oder Geschwistern vermehrt an der frischen Luft am Joggen oder Fahrrad fahren. Ein positiver Effekt ist, dass Menschen teilweise neu begonnen haben $\mathrm{zu}$ walken, zu joggen und Rad zu fahren. Zum anderen haben die digitalen Angebote vor dem Bildschirm zu Hause enorm an Zuwachs gewonnen. In der Konsequenz scheinen sich Heranwachsende trotz der vielen kurzzeitig verschlossenen Türen des organisierten Sports sogar mehr als vor der Covid-19-Pandemie zu bewe- gen. Auch wenn der organisierte Sport wieder allmählich stattfindet, können Mannschaftssportarten nur bedingt ausgeübt werden. Es dominieren aktuell Individualsportarten, vor allem beim informellen Sport.

Die MoMo-Studie zeigte letztes Jahr, dass nur $15 \%$ der Kinder und Jugendlichen die Empfehlung der WHO für körperliche Aktivität (mindestens eine Stunde mit mittlerer Intensität) erfüllen (vgl. Woll et al. 2019). Während des außergewöhnlichen Lebensabschnitts der Corona-Pandemie erfüllen nun $31 \%$ der Heranwachsenden ein Mindestmaß an Bewegung (vgl. Frenken et al. 2020). Dieser Anstieg in einer Teilstichprobe der großen MoMo-Kohorte wird auf den Mangel an alternativen Angeboten (zum Beispiel Nachhilfe, Musikschulunterricht) und die oben skizzierten zwei neuen Trends zurückgeführt. Gleichzeitig sind jedoch die neuesten Befunde der DAK Gesundheit alarmierend: Laut den Zwischenergebnissen der Studie „Mediensucht 2020“ sind die Nutzungszeiten für Gaming werktags um $75 \%$ im Vergleich zu den Werten vor der Corona-Krise erhöht (vgl. DAK-Gesundheit 2020, S. 2).

Was weiß die Wissenschaft bis jetzt, welche Befunde kennen Sie zu den Auswirkungen der Corona-Krise? Insgesamt stehen wir noch in den Anfängen. Wissenschaft benötigt Zeit, um Erkenntnisse $\mathrm{zu}$ generieren. Während einige Heranwachsende und Erwachsene sich inzwischen vielleicht sogar mehr bewegen, hat der Bewegungsmangel bei einem nicht unerheblichen Teil der Menschen sicherlich zugenommen. Erkenntnisse aus der Raumfahrt und aus Krankenhaus-Studien zeigen: Wenn ein Mensch drei Wochen am Stück vollkommen inaktiv ist, verliert er die Hälfte seiner Muskelkraft und büßt enorm an Ausdauervolumen ein (vgl. Woll 2020b). Bewegungsmangel zeigt also schnell Auswirkungen. Erheblich ist dabei, dass Bewegungsmangel und ein geringer familiärer Sozialstatus korrelieren - eine Entwicklung, die sich unter den Voraussetzungen einer solchen Krise durchaus verstärken könnte: Bewegung ist nämlich zentral abhängig von sportlichen 


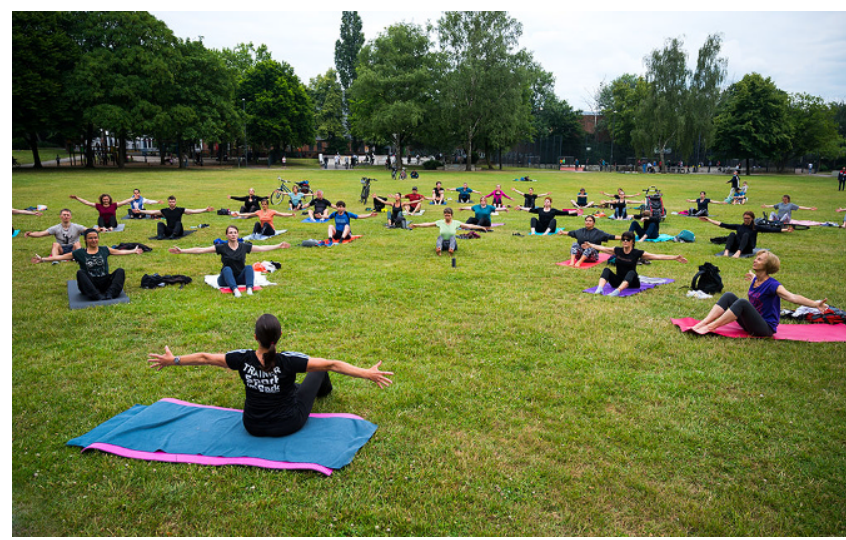

Abb. $2 \triangleleft$ Eine besondere Bedeutung könnte die Gestaltung von öffentlichen Plätzen für informellen Sport einnehmen. (Foto: LSB NRW/Andrea Bowinkelmann)

Vorbildern (Eltern und Geschwister), bewegungsfreudigen Freund ${ }^{\star}$ innen und Bewegungsmöglichkeiten in der näheren Umgebung (zum Beispiel Bewegungsmöglichkeiten im Garten oder auf dem Spielplatz). Es könnte gut sein, dass sich die soziale Schere während der Corona-Krise nun noch deutlicher auf den Bewegungsstatus auswirkt.

Welchen Bedarf an Forschung sehen Sie, wo sollten Schwerpunkte gesetzt werden und welche Ansätze ergeben sich für Sie persönlich aus der Pandemie? Im Moment erleben wir eine Veränderung der Sportkultur in Deutschland. Das Risiko einer zweiten Welle kann nicht ausgeschlossen werden und ein „normaler" organisierter Sport scheint auch mittelfristig nicht möglich. Interessant ist es, nach Möglichkeiten für Sportangebote für bislang sportabstinente Gruppen $\mathrm{zu}$ suchen, die eine (sportliche) Betreuung aufgrund der Situation im Elternhaus besonders nötig haben. Eine besondere Bedeutung könnte dabei die Gestaltung von öffentlichen Plätzen für den informellen Sport (• Abb. 2) oder das Gewährleisten digitaler Angebote einnehmen. Hier wären die Bedürfnisse zu ermitteln, also welche Raum- und (digitalen) Sportbedarfe diese und weitere Zielgruppen aufweisen. Auffällig bei den digitalen Sportangeboten ist, dass diese sich in ihrer Qualität teilweise enorm unterscheiden. Wichtig fände ich es so etwas wie Qualitätsstandards zu entwickeln. Darüber hinaus leiden im Moment aufgrund der Abstandsbestimmungen viele soziale Kontakte im Sport. Sport ist im
Moment vor allem Individualsport. Da stellt sich mir die Frage, inwiefern dies kurz- und langfristig Auswirkungen auf die zwischenmenschlichen Beziehungen haben kann.

Zeitpunkt der Antwort: 31. August 2020.

\section{„Informelles Sporttreiben begünstigt"}

\section{Forschungsthema: Interkulturelles} Lernen im Kinder- und Jugendsport

\section{Prof. Dr. Petra Gieß-Stüber}

Welche Auswirkungen hat die Covid19-Pandemie auf das Aufwachsen und das Sportengagement von Kindern und Jugendlichen aus Ihrer Forschungsperspektive? Insbesondere Kinder und Jugendliche mit schwierigen ökonomischen und sozialen Lebensbedingungen sind gefährdet, den Zugang zu Sport zu verlieren und damit von gesundheitsbezogenen und sozialen Funktionen nicht profitieren zu können:

- Sportliche (In-)Aktivität kann als familiäres Erbe verstanden werden. So werden die jungen Menschen nicht von der Familie zu körperlicher Aktivität motiviert und konnten nicht an der Schule beziehungsweise über Schulsport-AGs angesprochen werden.

- Die temporäre Aussetzung des Vereinssports hat für viele Menschen informelles Sporttreiben begünstigt. Aufgrund fehlender Einbindung in sportaktive Peergroups ist dieser Ersatz für eher sportdistanzierte Kinder nicht gegeben.

- Digitale Sportangebote setzen entsprechende Endgeräte in Haushalten voraus. Diese sind in manchen Familien schon für die Schulbildung nicht ausreichend vorhanden.

- Sportvereine konzentrieren sich in und nach der Krise auf die Reaktivierung vor allem des wettkampforientierten Spielbetriebs. Ressourcen werden voraussichtlich (noch) weniger für sozialgesellschaftliche Aufgaben des Sports eingesetzt.

Durch das Gebot sozialer Distanzierung fallen wertvolle Gelegenheiten persönlicher Begegnungen zwischen Kindern und Jugendlichen unterschiedlicher sozialer und kultureller Herkunft weg (- Abb. 3). Gemeinsames Sporttreiben als Vehikel für gesellschaftlichen Zusammenhalt ist deutlich erschwert.

Welchen Bedarf an Forschung sehen Sie, wo sollten Schwerpunkte gesetzt werden und welche Ansätze ergeben sich für Sie persönlich aus der Pandemie? Mich interessiert vor allem diese Frage: Wie könnte eine diversitätssensible kommunale Sportversorgung aussehen, die konsequent für alle Bürger ${ }^{\star}$ innen adäquate - auch niedrigschwellig zugängliche - Angebote bereithält?

Zeitpunkt der Antwort: 2. September 2020.

\section{„Pädagogischer Optimismus"}

\section{Forschungsthema: Qualifikation im Kinder- und Jugendsport}

Prof. Dr. Detlef Kuhlmann

Welche Auswirkungen hat die Covid19-Pandemie auf das Aufwachsen und das Sportengagement von Kindern und Jugendlichen aus Ihrer Forschungsperspektive? Die Pandemie hat uns alle im Sport unvorbereitet getroffen. So gesehen gab es keine Präventionsmaßnahmen, auch nicht, was das Sportengagement von Kindern und Jugendlichen angeht. 


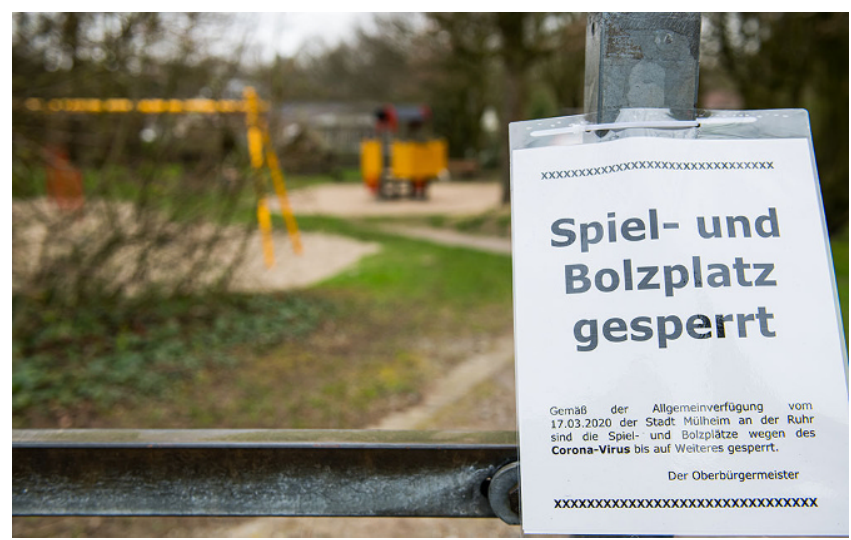

Abb. $3 \Delta$ Durch das Gebot sozialer Distanzierung fallen wertvolle Gelegenheiten persönlicher Begegnungen zwischen Kindern und Jugendlichen unterschiedlicher sozialer und kultureller Herkunft weg. (Foto: LSBNRW/ Andrea Bowinkelmann)

Es kam plötzlich über Monate zum völligen Time-out mit Stillstand beim Sportunterricht in der Schule und im Sportverein. So etwas gab es noch nie. Insofern ist es sicher schwierig, zum jetzigen Zeitpunkt alle Auswirkungen exakt zu benennen, geschweige denn die Folgen, die sich daraus ergaben und immer noch ergeben, in Art, Umfang und Intensität einzuschätzen. Nur zwei Beispiele: Wenn es über einen längeren Zeitraum keine Möglichkeit gibt, Schwimmen unter Anleitung zum Beispiel in einem Kurs zu lernen, dann kann das sowohl zur Folge haben, dass es später zu einem Kapazitätsstau kommt als auch dass Kinder das Schwimmen gar nicht oder anderswie lernen (• Abb.4). Zweites Beispiel: Wenn zum Beispiel der Trainings- und Wettkampfbetrieb in einer Ballspielsportart über längeren Zeitraum nicht stattfindet, muss das in Zeiten von Corona nicht zwangsläufig $\mathrm{zu}$ Abmeldungen vom Verein führen - zumal, wenn es kaum Alternativen durch Ausweichen in andere Sportarten gibt. Aber Fakt ist auch, es kommen keine neuen Kinder und Jugendlichen mehr hinzu, wenn der Sportverein über längere Zeit geschlossen ist. Dennoch oder gerade deswegen möchte ich gern ein wenig pädagogischen Optimismus streuen. Wir hören immer wieder: „Der Sport und damit auch der Kinder- und Jugendsport muss gestärkt aus der Krise zurückkehren!“ Das mag banal und bieder klingen, ist aber durchaus als Aufforderung gemeint, auch und gerade im

Sport(-verein) irre Ideen und innovative Formate zu entwickeln, um Kinder und Jugendliche (wieder) zu erreichen und neu zu begeistern.

Was weiß die Wissenschaft bis jetzt, welche Befunde kennen Sie zu den Auswirkungen der Corona-Krise? Wenn ich die Frage wörtlich nehme, muss ich passen: Woher soll ich wissen, was „die Wissenschaft“ weiß ... aber im Ernst: Bei einer Vielzahl von aktuellen Studien mit unterschiedlichen Fragestellungen aus verschiedenen Wissenschaftsdisziplinen müssen wir deren Befunde auch immer konstruktiv-kritisch interpretieren - hierzu ebenfalls ein Beispiel: Die Corona-Krise war rund sechs Wochen alt, da wurden erste Ergebnisse einer großen „kurzfristig organisierten welt- weiten Studie" mit angeblich 15.000 Probanden aus 52 Ländern vorgelegt. Dabei sollte herausgefunden werden, wie sich das Sport- und Bewegungsverhalten der Menschen in Zeiten von Corona verändert und welche Auswirkungen dies auf das subjektive Wohlbefinden hat. Ob dabei auch Jugendliche aus den 52 Ländern befragt wurden? Egal - im Ergebnis zeigt sich, dass Menschen, die sich schon vor der Corona-Krise regelmäßig bewegt und Sport getrieben haben, während der Pandemie aktiver waren beziehungsweise sind als jene, die sich schon zuvor nicht oder nur unregelmäßig bewegt haben. Ist dieser Befund überraschend? Gilt er dann auch für Kinder- und Jugendliche, selbst wenn sie gar nicht dazu befragt wurden? Wie dem auch sei: Die Autoren setzen sich 




Abb. $5<$ Den Wissenschaftler Nils Neuber „,interessiert vor allem, wie Kinderund Jugendliche die Ausnahmesituation erleben". (Foto: LSB NRW/Andrea Bowinkelmann) für weitere Lockerungen der bisherigen behördlichen Einschränkungen ein und machen sich „für ein gesundheitsdienliches Sporttreiben“" stark. Dem wird wohl niemand per se widersprechen.

\section{Welchen Bedarf an Forschung sehen Sie, wo sollten Schwerpunkte gesetzt werden und welche Ansätze ergeben sich für Sie persönlich aus der Pande- mie? Ich beschränke mich auf Teil drei der Frage und greife hier einen ganz spe- identitätsstiftenden Kern unseres Faches für die Lehre neu definieren, sondern uns auch fragen, welche soziale Identität wir den jungen Menschen bieten, die ein Studium - egal in welchem Studienfach - an einer Hochschule aufnehmen.} ziellen Aspekt auf, der mich in meiner derzeitigen (ehrenamtlichen) Funktion als Vorsitzender des Fakultätentages Sportwissenschaft sehr beschäftigt. Der Fakultätentag ist der Zusammenschluss aller rund 60 Fakultäten und Institute für Sportwissenschaft an den Universitäten und Pädagogischen Hochschulen in Deutschland. Wir haben gerade unser erstes „Online-Semester" hinter uns, was für uns alle eine neue Herausforderung war. Aber für unser Fach kommt hinzu: Das Studium besteht $\mathrm{zu}$ einem nicht geringen Anteil aus sogenannten Praxisveranstaltungen (zum Beispiel Leichtathletik, Ballspiele, Wassersport), die unter Corona-Bedingungen umorganisiert werden mussten oder gar nicht stattfinden konnten. Eine in Teilen vergleichbare Situation haben wir im letzten Schuljahr auch für den Sportunterricht erlebt. Man mag das alles für eine „notwendige“ Ausnahme halten - aber: Die digitalen Anteile in der (hoch-)schulischen Lehre werden so oder so weiter ausgebaut. Was hat das für Konsequenzen für unser Fach beziehungsweise für den Schulsport? Nur als ein Szenario so viel: Wenn immer mehr Lehrveranstaltungen an den Unis online angeboten werden, braucht man zukünftig immer wenige Hörsäle und Seminarräume. Wozu noch Mensen? Warum noch freiwilliger Hochschulsport für alle Studierenden? Am Ende müssen wir nicht nur den

Zeitpunkt der Antwort: 23. Juli 2020.

\section{„Schulfixiertes Bildungs- verständnis der Politik“}

\section{Forschungsthema: Informelles Lernen im Sport}

\section{Prof. Dr. Nils Neuber}

Welche Auswirkungen hat die Covid19-Pandemie auf das Aufwachsen und das Sportengagement von Kindern und Jugendlichen aus Ihrer Forschungsperspektive? Die Corona-Pandemie hat für Kinder und Jugendliche zunächst dieselben Auswirkungen wie für alle Menschen: Verlust vertrauter Umgebungen und Routinen, Abnahme von sozialen Interaktionen, Unsicherheit und Angst in Bezug auf die Krankheit ... Je nach Alter und Entwicklungsstand können Heranwachsende die Bedeutung der weltweiten Bedrohung unterschiedlich gut nachvollziehen. Hier sind wir Erwachsenen gefordert, eine angemessene „Übersetzung“ zu liefern. Die öffentli- che Wahrnehmung hat sich sehr stark auf das schulische Lernen und hier die Kernfächer konzentriert. Das ist einerseits zunächst nachvollziehbar, andererseits sind Kinder und Jugendliche keine „Lernmaschinen“. Informelle Tätigkeiten, wie das Spielen mit anderen Kindern, das Treffen mit Gleichaltrigen, das gemeinsame „Abhängen“, gehören genauso zum Aufwachsen dazu wie gemeinsame Bewegungs- und Sportaktivitäten. Und diese Tätigkeiten sind mindestens genauso entwicklungsrelevant wie Mathematik- und Deutschunterricht. Für mich war es erschreckend, wie einseitig schulfixiert sich das Bildungsverständnis von Bildungspolitikern in der CoronaPandemie äußerte. Das zeigt, dass hier noch viel Nachholbedarf besteht!

\section{Was weiß die Wissenschaft bis jetzt,} welche Befunde kennen Sie zu den Auswirkungen der Corona-Krise? Neben zahlreichen medizinischen Studien, die die Auswirkungen des coronabedingten Bewegungsmangels untersuchen, gibt es auch einige sozialwissenschaftliche Studien. So haben Langmeyer und andere untersucht, wie sich Kindsein in Zeiten von Corona verändert hat, zum Beispiel haben Drinnen-Spielen, Basteln, Malen und Lesen, aber auch Fernsehen, Computerspielen und Internetkonsum deutlich zugenommen (Langmeyer et al. 2020). Andresen und Kollegen haben die Erfahrungen von Jugendlichen untersucht und stellen neben Einsamkeitsgefühlen und Verunsicherung auch eine Reduktion junger Menschen auf das Funktionieren als Schüler ${ }^{\star}$ in fest. Ein Jugendlicher beschreibt das so: „Wir Jugendlichen werden doch nur als Schüler gesehen. Wir sollen lernen und lernen und lernen. Warum wird darüber diskutiert, die Sommerferien zu kürzen? Politiker denken wie Kapitalisten“ (Andresen et al. 2020a). Ein weiteres Thema ist die $\mathrm{Zu}$ nahme sozialer Benachteiligung durch die Corona-Pandemie. Während Heranwachsende aus gut situierten Familien auf zahlreiche Unterstützungsangebote zurückgreifen können, sind junge Menschen aus unteren sozialen Milieus häufig auf sich allein gestellt. Im Deutschen Schulbarometer gehen beispielsweise $86 \%$ der Lehrkräfte davon 




Abb. $6<$ Die pädagogische Qualität des Trainings im Kindersport hängt stark von persönlichen Kompetenzen der Lehrkräfte $\mathrm{ab}$ : Engagierte Trainer*innen werden diese Kompetenzen auch unter veränderten Bedingungen fruchtbar einbringen können. (Foto: LSB NRW/ Marc Hermenau)

aus, dass sich die soziale Ungleichheit durch die Schulschließung verstärken wird (Robert Bosch Stiftung $\mathrm{GmbH}$ 2020).

\section{Welchen Bedarf an Forschung sehen} Sie, wo sollten Schwerpunkte gesetzt werden und welche Ansätze ergeben sich für Sie persönlich aus der Pandemie? Mich persönlich interessiert vor allem, wie Kinder und Jugendliche die Ausnahmesituation erleben (• Abb. 5) - und was wir tun können, um die Folgen weiterer Einschränkungen für sie möglichst gering zu halten: Wie können wir soziale Interaktionen trotz Abstandsregeln ermöglichen? Welche digitalen oder analogen Bewegungsangebote können trotz Einschränkungen pädagogisch sinnvolle Impulse über ein reines Fitnesstraining hinaus setzen? Welche Formen der Beteiligung sind möglich, auch wenn Klassen oder Vereinsmannschaften nur digital zu- sammenkommen? Damit verbunden ist die Frage nach sich verändernden Körperverständnissen unter Corona-Bedingungen. Eine aktuelle These besagt, dass die Gesellschaft durch die zunehmende Digitalisierung auch vor Corona schon „körperloser“ geworden ist - stimmt das, oder gehen junge Menschen nur anders mit ihrer Körperlichkeit um? Schließlich fände ich spannend, das Potenzial informeller Lernprozesse unter Coronabedingungen auszuloten. Wenn weniger formale Lernprozesse über Lehrkräfte in der Schule stattfinden - findet dann automatisch kein Lernen statt? Oder organisieren Heranwachsende ihr Lernen dann neu? Steckt darin vielleicht auch eine Chance für die Nach-CoronaZeit? Einige Schulen planen unter dem Label „Frei-Day for Future“ schon mit verstärktem Projektunterricht.

Zeitpunkt der Antwort: 9. August 2020.

\section{„Beziehung und Interaktion bleiben zentrale Faktoren"}

\section{Forschungsthema: Pädagogik im Kinderleistungssport}

\section{Prof. Dr. Alfred Richartz}

Welche Auswirkungen hat die Covid19-Pandemie auf das Aufwachsen und das Sportengagement von Kindern und Jugendlichen aus Ihrer Forschungsperspektive? Aus meiner Sicht kann man zu mittel- und langfristigen Auswirkungen bislang nur spekulieren. Das erscheint mir nur in Grenzen nützlich. Die pädagogische Qualität des Trainings im Kindersport hängt stark von persönlichen Kompetenzen der Lehrkräfte ab (• Abb. 6): Wie gut gelingt es $\operatorname{der}^{\star}$ dem Trainer ${ }^{\star}$ in, unterstützende, persönliche Beziehungen und ein positives Trainingsklima zu entwickeln, wie effektiv werden die Trainingsabläufe gesteuert und wie gut ist die Qualität von Instruktion und Feedback? Engagierte Trainer*innen werden diese Kompetenzen auch unter veränderten Trainingsbedingungen und unter Verwendung neuer Medien und Methoden fruchtbar einbringen können. Denn auch bei der Nutzung digitaler Kommunikationsmedien und der Einschränkung des direkten persönlichen Kontakts bleiben Beziehung und Interaktion zentrale Faktoren für Lernen, Motivation und Wohlbefinden.

Was weiß die Wissenschaft bis jetzt, welche Befunde kennen Sie zu den Auswirkungen der Corona-Krise? Dazu kann ich für meinen Forschungsschwerpunkt noch nichts sagen. Pädagogisch relevante Faktoren ändern sich schnell (Präsenztermine, Gruppengröße und so weiter).

Welchen Bedarf an Forschung sehen Sie, wo sollten Schwerpunkte gesetzt werden und welche Ansätze ergeben sich für Sie persönlich aus der Pandemie? Man muss sehen, welche Veränderungen sich dauerhaft etablieren und Forschungsfragen daran ausrichten. Ich habe bereits vor der Pandemie ein Forschungsprojekt zur Förderung der pädagogischen Trainingsqualität durch 


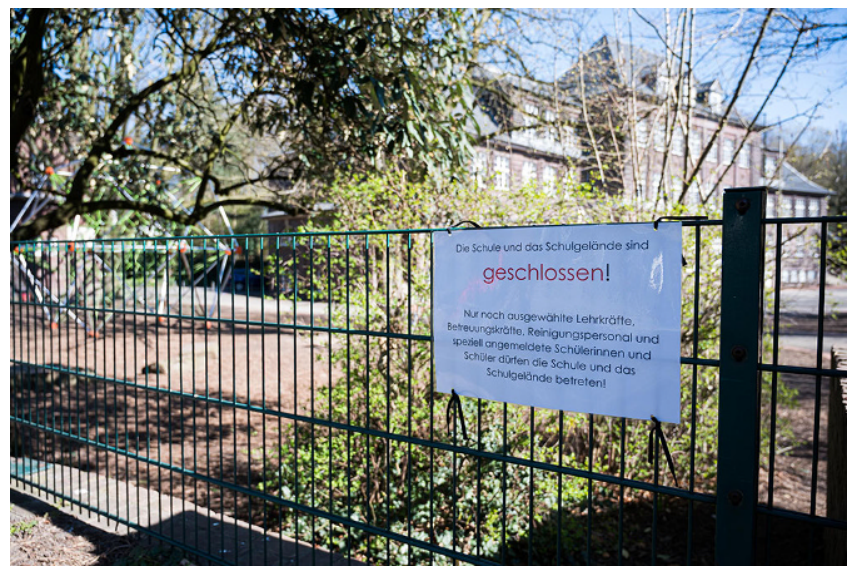

Abb. $7 \Delta$ Sind Präsenzunterricht in der Schule, Kontakte zu Gleichaltrigen, Sport im Verein oder der Besuch eines Jugendtreffs nur eingeschränkt möglich oder fallen gar komplett weg, fehlen jungen Menschen Möglichkeiten, sich Unterstützung bei Problemen in der Familie zu holen. (Foto: LSBNRW/ Andrea Bowinkelmann)

Online-Coaching von Trainer*innen durchgeführt und bin auch jetzt mitten in einem solchen Projekt. Geändert hat sich, dass die Plattform Zoom, die wir seit 2016 benutzen, jetzt weithin bekannt ist. Auch in der Trainer*innen-Fortbildung war ich jetzt bereits mehrfach in online-basierten Lehrgangsformen als Referent tätig. Solche Lehrgangsformate gab es schon vor der Pandemie, sie werden sich möglicherweise nun schneller weiterverbreiten. Aber die damit verbundenen Forschungsfragen sind nicht brandneu: Was wirkt? Wie kann man über Wissensvermittlung hinaus das pädagogische Können fördern? Und ähnliches mehr.

Zeitpunkt der Antwort: 7. September 2020.

\section{„Was bedeutet dies für vulnerable Kinder?"}

\section{Forschungsthema: Prävention sexualisierter Gewalt im Kinder- und Jugendsport}

\section{Prof. Dr. Bettina Rulofs}

Welche Auswirkungen hat die Covid19-Pandemie auf das Aufwachsen und das Sportengagement von Kindern und Jugendlichen aus Ihrer Forschungsperspektive? Kinder und Jugendliche sind durch die Kontaktbeschränkungen und soziale Distanzierung stark auf ihre Fa- milien begrenzt - ganz besonders in der Phase eines Lockdowns oder einer Schulschließung. Der Lockdown und die nachfolgenden Einschränkungen haben für einige Eltern und Familien zu äußerst belastenden Situationen geführt, wenn durch Einkommenswegfall oder Kurzarbeit finanzielle Engpässe entstehen oder Kinder rund um die Uhr zu Hause betreut werden müssen und gleichzeitig die Arbeit ruft.

Aus der Forschung zu Gewalt gegen Kinder ist allgemein bekannt, dass junge Menschen Missbrauch und insbesondere sexualisierte Gewalt am häufigsten im Kontext der Familie erleben. Untersuchungen zeigen zudem einen deutlichen Anstieg der häuslichen Gewalt zwischen Erwachsenen in Phasen der wirtschaftlichen Rezession (Schneider et al. 2016). Es liegt somit nahe zu befürchten, dass Kinder gerade in Phasen einer Krise, die mit Kontaktbeschränkungen einhergeht, besonders gefährdet sind, innerfamiliäre Gewalt zu erleben - als Opfer oder als Beobachtende von Gewalt. Wenn Präsenzunterricht in der Schule, Kontakte zu Gleichaltrigen, Sport im Verein oder der Besuch eines Jugendtreffs nur eingeschränkt möglich sind oder gar komplett wegfallen, fehlen für junge Menschen somit auch Möglichkeiten, sich Unterstützung bei Problemen und Gewalt in der Familie zu holen beziehungsweise sich zumindest zeitweise davon zu entlasten (•Abb. 7).
Was weiß die Wissenschaft bis jetzt, welche Befunde kennen Sie zu den Auswirkungen der Corona-Krise? Bis dato liegen noch nicht ausreichend differenzierte Daten vor, um das Ausmaß von Risiken und Kindeswohlgefährdungen unter den Bedingungen der Pandemie genau abzuschätzen. Eine Studie des Deutschen Jugendinstituts zeigt zum Beispiel, dass rund die Hälfte der befragten Jugendämter angeben, die Zahl der Gefährdungsmeldungen sei im Zeitraum vom 23. April bis 12. Mai 2020 in etwa gleich geblieben und ein weiteres Viertel der Jugendämter gibt an, die Zahl der Gefährdungsmeldungen sei in diesem Zeitraum gesunken. Diese Befunde können vorsichtig so gedeutet werden, dass Kindeswohlgefährdungen während des Lockdowns seltener von den Sozialdiensten entdeckt werden, da die Kommunikationswege von Schulen, Kitas und anderen Trägern der Jugendarbeit in Richtung der Jugendämter wegfallen (Mairhofer et al. 2020).

Die bundesweite Studie KiCo über die Erfahrungen von Eltern und Kindern während der Corona-Pandemie zeigt ein geteiltes Bild der Familien im Hinblick auf die Bewältigung der Belastungen unter den Bedingungen der Krise. Während einige Familien mit der neuen Situation gut zurechtkommen und diese sogar als Chance sehen, in der Familie neue Ressourcen zu schöpfen, zeigt sich ein Teil der Familien als stark belastet: Ein Drittel der befragten Eltern berichtet von größeren Geldsorgen während der Pandemie und immerhin $60 \%$ der Eltern fühlen sich mit ihren Sorgen nicht gehört und etwa von den Verantwortlichen in der Politik allein gelassen (Andresen et al. 2020b). Die vorliegenden Daten verweisen somit auf gestiegene Belastungen für einen Teil der Familien während der CoronaPandemie. Dass dies auch zu einem $\mathrm{Zu}$ wachs an innerfamiliären Konflikten und häuslicher Gewalt führt, ist zu vermuten, muss jedoch zukünftig noch durch weitere Studien differenziert belegt werden.

Welchen Bedarf an Forschung sehen Sie, wo sollten Schwerpunkte gesetzt werden und welche Ansätze ergeben sich für Sie persönlich aus der Pandemie? Gänzlich ungeklärt ist bislang noch 




Abb. $8<$ Forschungsbedarf wird gesehen bei der Entwicklung digitaler Werkzeuge für die Sportorganisationen und für den Schulsport, etwa in Bezug auf Fort- und Weiterbildung. (Foto: LSB NRW/Andrea Bowinkelmann)

die Frage, welche Bedeutung Sportvereine für den Schutz von Kindern haben können, wenn sie in ihren Familien Konflikte oder Gewalt erfahren. In meinen eigenen Studien zu Gewalt habe ich bisher schwerpunktmäßig untersucht, wie häufig und unter welchen Bedingungen Kindesmissbrauch und Gewalt im Sport geschehen und welche Schutzmaßnahmen es in Sportverbänden und -vereinen gibt. Diese Daten zu erheben war wichtig und lange überfällig. Allerdings gerät dabei noch zu wenig in den Blick, dass Sportvereine für junge Menschen auch Orte der Zuflucht, Unterstützung und des Empowerments sein könnten - vielleicht gerade oder besonders dann, wenn Kinder und Jugendliche in ihren Familien nicht ausreichend Schutz erfahren. Sport im Verein zu treiben war und ist unter den Bedingungen der Pandemie gar nicht oder nur eingeschränkt möglich. Was bedeutet dies insbesondere für die sogenannten vulnerablen Kinder, die in ihren Familien starken Belastungen oder Gewalt ausgesetzt sind? Inwiefern sind Sportvereine und die darin tätigen Trainer*innen gerade für diese Kinder wichtige Anlaufstellen? Wie können sich Sportvereine mit anderen Institutionen der Kinder- und Jugendarbeit in ihren Kommunen vernetzen, um den Schutz von Kindern zu verbessern? Diese Fragen gilt es grundsätzlich - und besonders in Zeiten einer gesellschaftlichen Krise - zu untersuchen.

Zeitpunkt der Antwort: 8. September 2020.

\section{„Forschungsbedarf in der Entwicklung digitaler Tools"}

\section{Forschungsthema: Kommunale Kinder- und Jugendsport- entwicklung}

\section{Prof. Dr. Jessica Süßenbach}

Welche Auswirkungen hat die Covid19-Pandemie auf das Aufwachsen und das Sportengagement von Kindern und Jugendlichen aus Ihrer Forschungsperspektive? Der Schulsport leistet einen wichtigen Beitrag zu einer allseitigen Bildung und Erziehung von Kindern und Jugendlichen. Dies gilt sowohl für den Sportunterricht als auch für die außerunterrichtlichen Angebote, die im Ganztag von Übungsleiter*innen und Trainer*innen der Sportvereine als auch von freien Honorarkräften geleistet werden. Der Deutsche Olympische Sportbund (DOSB) und die Deutsche Sportjugend (dsj) bieten dazu auch aktuell ausdrücklich ihre Kooperation an. Auf der einen Seite sind die zahlreichen kreativen Sportangebote in und mit digitalen Medien (zum Beispiel Albas tägliche Sportstunde) hervorzuheben, die häufig von Sportlehrkräften im Online-Unterricht (soweit vorhanden) eingesetzt wurden und so Eingang in den Schulsport finden. Andererseits sind durchaus berechtigte Mahnungen und Forderungen aus dem organsierten Sport zu vernehmen, die auf die Sicherung der Strukturen der Kinder- und Jugendarbeit verweisen, um die Angebote des Kinder- und Jugendsports aufrechterhalten zu können (zum Beispiel die Beschäftigung der Freiwilligendienstleistenden).

Zentral ist meines Erachtens die bildungspolitische Erkenntnis, dass insbesondere Heranwachsende aus benachteiligten Milieus in der neuen „Normalität“ weiter abgehängt werden und dementsprechend mit Blick auf die Sport- und Bewegungsangebote in formalen (Schule) und non-formalen (Sportvereine) Settings „unterversorgt“ bleiben.

Was weiß die Wissenschaft bis jetzt, welche Befunde kennen Sie zu den Auswirkungen der Corona-Krise? Hier möchte ich auf zwei Befunde aus der ifoStudie vom August 2020 verweisen. Einmal zur Nutzung der freigewordenen Zeit während der Schulschließungen: „Die Corona-bedingten Schulschließungen haben zu einer deutlichen Verringerung der Lernzeit geführt. So hat sich die Zeit, die Schulkinder mit schulischen Aktivitäten (Schulbesuch oder Lernen für die Schule) verbracht haben, von täglich $7,4 \mathrm{~h}$ vor Corona auf $3,6 \mathrm{~h}$ während Corona halbiert. Die freigewordene Zeit wurde nur in geringem Maße für zusätzliche entwicklungsförderliche Tätigkeiten wie Lesen, Musizieren oder Bewegung genutzt. Stattdessen haben die Schüler*innen vor allem mehr Zeit mit passiven Tätigkeiten wie Fernsehen, Computer- und Handyspielen oder sozialen Medien verbracht. Die tägliche Stundenzahl dieser Aktivitäten stieg von 4,0 vor Corona auf 5,2 während Corona. Nicht-Akademikerkinder und leistungsschwächere Schüler ${ }^{\star}$ innen verbrachten während der Schulschließungen besonders viel Zeit mit diesen Tätigkeiten, was befürchten lässt, dass die Coronakrise die Bildungsungleichheit in Deutschland verstärkt."

Eine andere Frage aus der ifo-Studie lautete: Welche Maßnahmen befürwortet die bundesdeutsche Bevölkerung? Hierzu heißt es: „Die deutsche Gesamtbevölkerung unterstützt verschiedene Maßnahmen, die helfen können, den Lernverlust durch die Schulschließungen möglichst gering zu halten. So spricht sich eine große Mehrheit dafür aus, Lehrkräfte während der Corona-bedingten Schulschließungen anzuweisen, täglich Kontakt mit ihren Schülerinnen zu halten. 


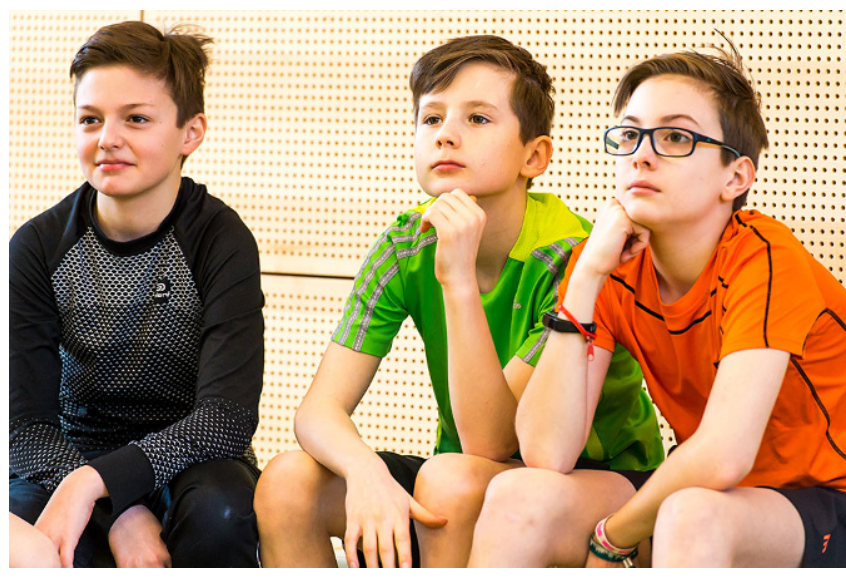

Abb. $9<$ Eine Alternative zu realem Sportunterricht könnten digitale Bildungsangebote sein, die auf eine kognitive Auseinandersetzung mit Sport und Bewegung abzielen. (Foto: LSB NRW/Andrea Bowinkelmann)

Ebenfalls finden sich deutliche Mehrheiten für die Vorschläge, dass Schulen bei Schulschließungen verpflichtend aufOnline-Unterricht umstellen müssen, dass Lehrkräfte sich zum Thema Online-Unterricht fortbilden müssen und dass Schüler*innen, deren Familien sich die technische Ausstattung nicht leisten können, mit Laptops ausgestattet werden. Auch die intensivere Betreuung von Kindern aus benachteiligten Verhältnissen während der Schulschließungen wird von klaren Mehrheiten befürwortet. Weniger einig sind sich die Deutschen darüber, welche Infektionsschutzmaßnahmen in Schulen nach den Schulöffnungen ergriffen werden sollen, aber auch hier finden sich Mehrheiten für verschiedene Vorschläge“ (Wößmann et al. 2020).

\section{Welchen Bedarf an Forschung sehen Sie, wo sollten Schwerpunkte gesetzt werden und welche Ansätze ergeben sich für Sie persönlich aus der Pande- mie? Zukünftige Forschungsperspekti- ven und -bedarfe sehe ich in der Ent- wicklung digitaler Tools für die Sport- organisationen und für den Schulsport. Diese Vorhaben sollten zum einen unter- richtliche Konzepte (in Bezug auf Fort- In diesem Forschungskontext startet so- bald wie möglich eine qualitative Studie zum Ganztagssport in Niedersachsen aus der Perspektive der Schüler*innen. \\ Zeitpunkt der Antwort: 7. September 2020. \\ "Das Unterrichtsfach Sport am Schluss des Fächerkanons"} und Weiterbildung, $\bullet$ Abb. 8) als auch sportbezogene Angebote und Vorhaben im schulischen Ganztag in den Blick nehmen. Empirisch fundierte Studien können sowohl den Einsatz digitaler Tools für die (barrierefreie) Teilhabe an Sport- und Bewegungsangeboten begründen als auch eine qualitative Verbesserung der Angebote bewirken. Aus sportpädagogischer Perspektive besteht meines Erachtens eine Notwendigkeit und disziplinäre Aufgabe darin, dem didaktisch mehr oder weniger reflektierten „Digitalisierungswahn“ konstruktiv $\mathrm{zu}$ begegnen. Derzeit arbeiten wir in zwei Forschungsprojekten zu diesem Themenfeld: erstens zum Medieneinsatz im Grundschulsport sowie zweitens zum digitalgestützen Üben im Fachunterricht in einem fachübergreifenden Verbundprojekt der Lehrer*innenbildung.

Darüber hinaus sollten gelingende Kooperationen zwischen Schule und Sportverein stärker in den Fokus der Forschung geraten. Trotz unterschiedlicher Handlungslogiken und in Teilen divergenter Zielvorstellungen könnte gerade jetzt eine Bündelung der Kräfte der Akteur*innen des Sports vor Ort helfen, (neue) Ideen und Konzepte für einen zeitgemäßen Schulsport zu entwickeln.

Forschungsthema: Persönlichkeitsund Teamentwicklung im Kinderund Jugendsport

Prof. Dr. Ralf Sygusch

Welche Auswirkungen hat die Covid19-Pandemie auf das Aufwachsen und das Sportengagement von Kindern und Jugendlichen aus Ihrer Forschungsper - spektive? Das Konzept zur Persönlichkeits- und Teamentwicklung sieht vor, dass Trainer*innen und Übungsleiter*innen im Trainings- und Wettkampfalltag gezielt und sportartspezifisch beispielsweise Selbstkonzept, Konzentrationsfähigkeit, Kooperationsfähigkeit und so weiter fördern können. Diese Förderung ist auf einen geregelten Trainings- und Wettkampfbetrieb ausgerichtet - und der kann so im Moment an vielen Orten nicht stattfinden. Solange der Sportbetrieb ruht, ist die Umsetzung der Grundidee, über Training und Bewegung im Sportverein die persönlichkeitswirksame Entwicklung zu unterstützen, auszulösen und anzuschieben, nicht möglich. Damit sind die angestrebten Potenziale und Wirkungen im formalen Bereich und non-formalen Bereich angesprochen. Alles, was an informellen Effekten und Wirkungen auf dieselben Parameter wie Selbstkonzept, soziale Kompetenz und so weiter wünschenswert ist, das kann ebenfalls nicht stattfinden. Insofern fehlen Kindern und Jugendlichen, wenn normaler Sport nicht möglich ist, entsprechende Entwicklungseinflüsse und entsprechende Lerngelegenheiten zur Persönlichkeitsentwicklung. Wissenschaft, Pädagogik oder Vereinsfunktionär*innen können in dieser Situation relativ wenig machen, außer abwarten und appellieren. Zumal mir die Virusentwicklung beziehungsweise deren Eindämmung im Großen und Ganzen bedeutsamer erscheint als die eine Trainingseinheit, die kooperatives Verhalten auslösen würde.

Was weiß die Wissenschaft bis jetzt, welche Befunde kennen Sie zu den Auswirkungen der Corona-Krise? Als Versuch einer Antwort kann ich etwas zum Schulsport sagen. In einem laufenden Forschungsprojekt haben wir massiv erlebt, dass das Unterrichtsfach Sport momentan ganz am Schluss des Fächerkanons zu stehen scheint. In dem Projekt haben wir das Thema Gesundheit im Sportunterricht in Schulen beziehungsweise in einzelnen Schulklassen über das gesamte Schuljahr begleitet und mit Interventionen unterstützt. Für die Intervention haben wir mit den Lehrkräften Unterrichts- 
einheiten entwickelt oder entwickeln wollen, die sich systematisch mit dem Thema Gesundheit auseinandersetzen. Beispielsweise hat eine Lehrkraft zum Thema Verletzungsrisiken im Judo eine Einheit entwickelt, in der es um das richtige Fallen geht. Das kann nicht nur im Judo, sondern auch den Sport- und Bewegungsalltag allgemein ein wichtiges, verletzungsprophylaktisches Thema sein. Die Schüler*innen hätten sozusagen eine Gesundheitskompetenz entwickelt, wie sie am Beispiel der Sportart Judo Verletzungen vorbeugen können. Eine andere Lehrkraft hat sich mit der Gestaltung von gesundheitsorientiertem Krafttraining befasst. Die Interventionen mit den Schüler*innen konnten aber wegen Corona nicht mehr stattfinden. Da ab Anfang des zweiten Schulhalbjahres kein Sportunterricht mehr stattgefunden hat, haben wir versucht, digitale Bildungsangebote $\mathrm{zu}$ schaffen. Was auf eine kognitive Auseinandersetzung mit Sport und Bewegung hinausläuft und dazu führen soll, Schüler*innen zum selbstständigen Sporttreiben zu befähigen (• Abb. 9). Interessant war die Reaktion der Schulen. Genau eine von vier Schulen hat gesagt: Da machen wir mit. Von den anderen kam die Rückmeldung, unter den jetzigen Bedingungen ganz grundsätzlich keinen Sportunterricht weiterentwickeln $\mathrm{zu}$ wollen und damit eben auch keine digitalen Bildungsangebote $\mathrm{zu}$ schaffen. Vor dem Hintergrund, dass Digitalisierung durch die Pandemie zum Megathema geworden ist, ist das schon bemerkenswert. Die eine Schule hat im Übrigen tatsächlich hochinteressante, sehr machbare digitale Unterrichtseinheiten zum Sportunterricht entworfen. Weiterhin interessant: Da wir im Rahmen dieses Projekts zu Beginn des letzten Schuljahres die Gesundheitskompetenz von Schüler*innen und deren sportliche Aktivität erfasst haben und das bis zum Ende des Schuljahres auch ohne Sportunterricht fortführten, hat sich quasi eine neue Fragestellung in das Projekt eingeschlichen: Ob die Schüler*innen, die zu Beginn des Schuljahres bereits eine höhere Gesundheitskompetenz besaßen, im Laufe der Pandemiezeit, in der kein Sportunterricht stattgefunden hat, ihr Aktivitätsverhalten anders aufrechterhielten als die Schüler*innen, die schon zu Beginn des Schuljahres eine sehr geringe Gesundheitskompetenz hatten. Das können wir im Idealfall jetzt statistisch ermitteln. Es gibt bereits Hinweise aus der Sportpsychologie, dass diejenigen, die sich mit Sport und dessen Bedeutung in ihrem Lebensalltag vor der Pandemie schon häufig auseinandergesetzt haben, auch diejenigen sind, die währenddessen eher aktiv geblieben sind.

Zeitpunkt der Antwort: 3. September 2020.

\section{Korrespondenzadresse}

\section{Oliver Kauer-Berk}

Redaktion Forum Kinder- und Jugendsport

Gelnhausen, Deutschland

okb@okb-redaktion.de

\section{Literatur}

Ammar, A., Trabelsi, K., Brach, M., Chtourou, H. (2020). Effects of home confinement on mental health and lifestyle behaviours during the COVID19 outbreak: insights from the ECLB-COVID19 multicentre study. Biology of Sport. https://doi. org/10.5114/biolsport.2020.96857.

Andresen, S., Lips, A., Möller, R., Rusack, T., Schröer, W., Thomas, S., \&Wilmes, J. (2020a). Erfahrungen und Perspektiven von jungen Menschen während der Corona-Maßnahmen. Erste Ergebnisse der bundesweiten Studie JuCo. Hildesheim: Hildesheim: Universitätsverlag. https://doi.org/10.18442/ 120.

Andresen, S., Lips, A., Möller, R., Rusack, T., Schröer, W., Thomas, S., \& Wilmes, J. (2020b). Kinder, Eltern und ihre Erfahrungen während der CoronaPandemie - Erste Ergebnisse der bundesweiten Studie KiCo. Hildesheim: Universitätsverlag Hildesheim.

Burrmann, U., Mutz, M., \& Braun, S. (2020). In whom do we trust? The level and radius of social trust among sport club members. International Review for the Sociology of Sport, 55(4), 416-436.

DAK-Gesundheit (Hrsg.). (2020). Mediensucht 2020 Gaming und Social Media in Zeiten von Corona: DAK-Längsschnittstudie: Befragung von Kindern, Jugendlichen (12-17 Jahre) und deren Eltern. Hamburg: Eigenverlag.

Frenken, S., Woll, A., \& Holze, K. (2020). Corona und Bewegungsmangel: „Wir brauchen eine Lobby für Kinder" (M. Schweizer). https://www.deutschlandfunk.de/corona-undbewegungsmangel-wir-brauchen-eine-lobbyfuer.1346.de.html?dram:article_id=477156 (Erstellt: 21. Mai 2020). Zugegriffen: 28. Juli 2020. Deutschlandfunk.

Guan, H., et al. (2020). Promoting healthy movement behaviours among children during the COVID19 pandemic. https://www.thelancet.com/ pdfs/journals/lanchi/PIIS2352-4642\%2820 \%2930131-0.pdf.Zugegriffen:3. Sept. 2020.
Langmeyer, A., Guglhör-Rudan, A., Naab, T., Urlen, M., \& Winklhofer, U. (2020). Kindsein in Zeiten von Corona. Erste Ergebnisse zum veränderten Alltag und zum Wohlbefinden von Kindern. Deutsches Jugendinstitut. https://www.dji.de/fileadmin/user_upload/ dasdji/themen/Familie/DJ__Kindsein_Corona Erste_Ergebnisse.pdf.Zugegriffen:2.Okt. 2020.

Mairhofer, A., Peucker, C., Pluto, L., van Santen, E., \& Seckinger, M. (2020). Kinder- und Jugendhilfe in Zeiten der Corona-Pandemie. DJlJugendhilfeb@rometer bei Jugendämtern. München: Deutsches Jugendinstitut. unter Mitarbeit von M. Gandlgruber

Mutz, M., \& Gerke, M. (2020). Sport and exercise in times of self-quarantine: How Germans changed their behaviour at the beginning of the Covid-19 pandemic. International Review for the Sociology of Sport. https://doi.org/10.1177/ 1012690220934335.

Robert Bosch Stiftung (2020). Das Deutsche Schulbarometer Spezial - Lehrerbefragung zur Corona-Krise. https://deutsches-schulportal. de/unterricht/das-deutsche-schulbarometerspezial-corona-krise/.Zugegriffen:2. Okt. 2020.

Schneider, D., Harknett, K., \& McLanahan, S. (2016). Intimate partner violence in the great recession. Demography, 53(2), 471-450.

Woll, A., Oriwol, D., Anedda, B., Burchartz, A., HanssenDoose, A., Kopp, M., Niessner, C., Schmidt, S., Bös, K., \& Worth, A. (2019). Körperliche Aktivität, motorische Leistungsfähigkeit und Gesundheit in Deutschland: Ergebnisse aus der Motorik-ModulLängsschnittstudie (MoMo). Karlsruhe: KIT.

Woll, A. (2020a). Corona und Bewegungsmangel. "Wir brauchen eine Lobby für Kinder". Interview im Deutschlandfunk. https:// www.deutschlandfunk.de/corona-undbewegungsmangel-wir-brauchen-eine-lobbyfuer.1346.de.html?dram:article_id=477156. Zugegriffen:3.Sept. 2020.

Woll, A. (2020b). Sportwissenschaftler zu Bewegung in Zeiten von Corona: „Ich plädiere für Sport-Hausaufgaben“ (M. Rieger). https://www. deutschlandfunk.de/sportwissenschaftler-zubewegung-in-zeiten-von-corona-ich.1346.de. html?dram:article_id=474983 (Erstellt: 19 . Apr. 2020). Zugegriffen: 28. Juli 2020. Deutschlandfunk.

Wößmann, L., Freundl, V., Grewenig, E., Lergetporer, P., Werner, K., \& Zierow, L. (2020). Bildung in der Coronakrise: Wie haben die Schulkinder die Zeit der Schulschließungen verbracht, und welche Bildungsmaßnahmen befürworten die Deutschen? ifo Institut, München, 2020. ifo Schnelldienst, 73(09), 1-17. 